\title{
Ambulatory Blood Pressure at Sea Level and High-altitude in a Climber with a Kidney \\ Transplant and Hypertension
}

Benoit Phelan, MD ${ }^{1}$, Luke Mather, $\mathrm{MD}^{2}$, Nirajan Regmi ${ }^{3}$, Jennifer Starling, $\mathrm{MD}^{4}$, David Twillman ${ }^{4}$, Matthew McElwee, MD ${ }^{5}$, Purshotam Paudel ${ }^{6}$, Buddha Basnyat ${ }^{3}$ and Linda E. Keyes, MD, FACEP .

${ }^{1}$ Dalhousie University ${ }^{2}$ University of Washington; ${ }^{3}$ Nepal International Clinic; ${ }^{4}$ University of Colorado;

${ }^{5}$ Case Western University; ${ }^{6}$ Tribhuvan University Teaching Hospital

Corresponding Author:

Linda E. Keyes, MD, FACEP, University of Colorado, Aurora, CO

Address: 3510 Endicott dr., Boulder, CO 803035

Email: Linda.Keyes@aya.yale.edu

Phone: 303-949-0963

\section{Word Count: 1790}

\section{Running Title: ABP in Kidney Transplant: Low vs. High Altitude}

Key words: kidney transplant, high altitude, hypertension, $24 \mathrm{~h}$ ambulatory blood pressure monitoring, immunosuppression, Nepal 


\begin{abstract}
Background and Objectives: High altitude may increase blood pressure (BP) and the kidney plays an important role in acclimatization. Little is known about how transplanted kidneys respond to the hypoxic stress of high altitude. We compared $24 \mathrm{~h}$ ambulatory blood pressure (24 ABP) in a climber with a kidney transplant and hypertension at sea level and high altitude (2800-4300m).

Methods: Welch-Allyn ABPM 6100 monitor was used to collect heart rate (HR), systolic BP (SBP), and diastolic BP (DBP) every 30 min while awake, and hourly while asleep. The kidney transplant participant was monitored for $49 \mathrm{~h}$ at sea level and for $53 \mathrm{~h}$ at 2800 $4300 \mathrm{~m}$.

Results: Overall mean SBP did not differ between altitudes. At high altitude the participant's mean nocturnal BP decreased, but this "reverse dipping” pattern was not observed at sea level. The participant had no evidence of altitude illness or infectious complication at high altitude.

Conclusions: This case builds on previous reports that kidney transplant recipients may safely travel to high altitude Further study is required to determine the generalizability to other travelers with kidney transplant and/or underlying hypertension and the clinical significance of short-term elevated nocturnal blood pressure at high altitude.
\end{abstract}




\section{Introduction}

With the increasing popularity of adventure travel and on going medical advancements, a growing number of individuals with comorbidities and polypharmacy are traveling to high altitude. Hypertension is a common disease in the general population as well as in high altitude trekkers(Keyes, Mather et al. 2016). Few studies have looked at individuals with chronic kidney disease or solid organ transplant at high altitude(Luks, Johnson et al. 2008, White, Touyz et al. 2009, Luks 2016). Whether or not these conditions predispose individuals to developing acute altitude illnesses or other undesirable medical events remains unclear.

The kidney plays an important role in acclimatization moderating volume status and erythropoietin production. (Goldfarb-Rumyantzev and Alper 2014). Prior investigations suggest that these functions should be preserved in renal transplant recipients(Luks 2016) though only a few reports have examined clinical outcomes of kidney transplant patients at high altitude (White, Touyz et al. 2009, Suh, Kim et al. 2015, van Adrichem, Siebelink et al. 2015) and none of these subjects had the complication of hypertension.

Travel to high altitude may influence blood pressure (BP) differently in normotensive and hypertensive individuals (Keyes, Sallade et al. 2017). Furthermore, the role and efficacy of antihypertensive medications in hypertensive individuals at altitude remains unclear(Luks 2009, Bilo, Caldara et al. 2011, Parati, Bilo et al. 2014).

We present a case study of a hypertensive renal transplant recipient at high altitude and compare his 24-hour ambulatory blood pressure (ABP) at sea level and between high altitude. 


\section{Case Presentation}

Our subject is a 57-year-old man who received a cadaveric kidney transplant in 2002 at age 45 years due to type 2 membranoproliferative glomerulonephritis and end-stage renal disease (ESRD). He progressively returned to mountain sports within 6 months of receiving his transplant and was back to mountaineering within 1 year. Since his transplant he has been above $6000 \mathrm{~m}$ on multiple occasions and has reached a maximal altitude of $6500 \mathrm{~m}$ without developing mountain sickness or other acute problems. His past medical history also includes hypertensive cardiomyopathy and mild valvulopathy. His medications include Losartan, Doxazosin, Mycophenolate Mofetil, Tacrolimus, Deflazacort and Allopurinol and did not differ between study periods at high altitude and at sea-level.

High-altitude ABP was measured in the Khumbu valley, Nepal when the participant enrolled as a participant in a prospective observational cohort study on BP, hypertension and high altitude in October 2014(Keyes, Sallade et al. 2017). Because of the limited information on kidney transplant patients at high altitude, we solicited the participant's consent to perform additional 24 ABPM at sea level for this report, and to share this data with his physicians. He gave written consent to participate in the larger study and for this case report.

Welch-Allyn ABPM 6100 monitor was used to collect heart rate (HR), systolic BP (SBP), and diastolic BP (DBP) every 30 min 07:00-22:00, and hourly 22:00-07:00. Summary high-altitude data has been partially reported separately(Keyes, Sallade et al. 2017). Here, we compare detailed ABP measurements during ascent from $2860 \mathrm{~m}$ to 
4300m with ABP measured at sea level in the south of Spain in January 2016. The participant wore the device for two consecutive days and recorded his daily activities. During high altitude ascent, acute mountain sickness was evaluated using the Lake Louise Score at 2860m, 3400m and 4300m.

\section{Results}

High altitude ascent

24-ABP was measured for a total of 53 hours during ascent from $2640 \mathrm{~m}$ to $4300 \mathrm{~m}$. The percentage of overall, awake and asleep BP values above American Heart Association (AHA) recommended normal cut-offs were $42 \%, 25 \%$ and $93 \%$ respectfully. Nocturnal BPs did not show normal dipping patterns. SBP and DBP rose $7 \%$ and $18 \%$, respectively. Blood pressure varied with activity but mean SBP and DBP remained similar across altitudes and showed no relationship with altitude (Table 1 and Fig 1).

The patient reported no symptoms of AMS at any altitude when these were assessed contemporaneously at 2800,3400 and $4400 \mathrm{~m}$. Patient denied any symptoms occurring during his ascent to $5000 \mathrm{~m}$ when questioned about this afterwards. He also denied symptoms of infection.

\section{Comparison between sea level and high altitude}

SL and HA ABP were measured for a total of 49 hrs and $53 \mathrm{hrs}$, respectively. Activity logs showed less sustained activity at SL, although strenuous physical activity was recorded on both days at sea level (data not shown). 
Mean overall and awake SBP were the same at SL and HA (table 1). At SL, fewer BP values were above AHA normal cut-offs compared to HA (overall 25.0\% vs. 41.9\%, awake $23.2 \%$ vs. $25.3 \%$ and asleep $31.3 \%$ vs. $92.9 \%$ respectively). However, the highest measured BP occurred at sea level, and maximum measurements at sea level were higher than maximum measurements at HA (table 1). There were no critically high SBP measurements $(>180 \mathrm{mmHg})$ at either altitude.

Mean nocturnal SBP and DBP were lower at SL (table 1). Overall DBP was higher at HA due to the increase in nocturnal DBP. At SL, nocturnal SBP and DBP decreased by $4 \%$ and $1 \%$, respectively. The observed decrease did not reach the dipping cutoff of $10 \%$, classifying the participant as "non-dipper", whereas at HA, nocturnal BP paradoxically rose above daytime values, classifying him as a "reverse-dipper" or "riser".

\section{Conclusions}

In this climber with a kidney transplant and hypertension overall mean SBP did not differ by altitude and maximal BP values occurred at SL. He did not develop AMS, suggesting his transplanted kidney was able to respond with appropriate diuresis and natriuresis to help him acclimatize(Luks 2016). In addition, despite immunosuppressant medication, he developed no serious infectious diseases during his trek.

Of note, the patient's nocturnal blood pressure pattern differed between SL and HA. Non-dipping and reverse-dipping tendencies are more common in patients with chronic kidney disease and in kidney transplant recipients compared to the normal population(Redon, Oliver et al. 1999, Velasquez, Beddhu et al. 2016). These tendencies are associated with worse long-term clinical outcomes(Muxfeldt, Cardoso et al. 2009, 
Wang, Ye et al. 2016) however, the short-term significance of this phenomenon at highaltitude is unknown. Our subject remained asymptomatic and demonstrated no adverse short-term effects and the reverse-dipping pattern resolved on return to sea-level. Whether longer HA exposure with persistent non-dipping or reverse dipping might have long-term adverse consequences is also unknown and may merit further investigation.

This case builds on previous reports that kidney transplant recipients may safely travel to high altitude (Suh, Kim et al. 2015, van Adrichem, Siebelink et al. 2015). Our case is unique in that the patient also had underlying hypertension and was older than the participants in previous reports. Despite his complicated medical history, our patient has been able to enjoy a successful high-altitude mountaineering career. Further study is required to determine the generalizability to other travelers with kidney transplant and/or underlying hypertension and the clinical significance of short-term elevated nocturnal blood pressure at high altitude.

\section{Figure Legend:}

Systolic (black) and diastolic (grey) blood pressure measurements over the study period at sea level and high altitude. Light grey areas designate sleep times.

\section{Acknowledgments}

This paper was funding in part by a Hultgren grant from the Wilderness Medical Society and the Nepal International Clinic. We thank Welch-Allyn for its generous donation of the $24 \mathrm{ABPM}$ monitor and our patient for his participation. 



\section{References}

Bilo, G., G. Caldara, K. Styczkiewicz, M. Revera, C. Lombardi, A. Giglio, A. Zambon, G. Corrao, A. Faini, M. Valentini, G. Mancia and G. Parati (2011). "Effects of selective and nonselective beta-blockade on 24-h ambulatory blood pressure under hypobaric hypoxia at altitude." L Hypertens 29(2): 380-387.

Goldfarb-Rumyantzev, A. S. and S. L. Alper (2014). "Short-term responses of the kidney to high altitude in mountain climbers." Nephrol Dial Transplant 29(3): 497506.

Keyes, L. E., L. Mather, C. Duke, N. Regmi, B. Phelan, S. Pant, J. Starling, M. McElwee, D. Cole, T. McConnell, P. Paudel, T. D. Sallade, A. Sheets, D. Twillman, D. S. Young and B. Basnyat (2016). "Older age, chronic medical conditions and polypharmacy in Himalayan trekkers in Nepal: an epidemiologic survey and case series." L Travel Med 23(6).

Keyes, L. E., T. D. Sallade, C. Duke, J. Starling, A. Sheets, S. Pant, D. S. Young, D. Twillman, N. Regmi, B. Phelan, P. Paudel, M. McElwee, L. Mather, D. Cole, T. McConnell and B. Basnyat (2017). "Blood Pressure and Altitude: An Observational Cohort Study of Hypertensive and Nonhypertensive Himalayan Trekkers in Nepal." High Alt Med Biol 18(3): 267-277.

Luks, A. M. (2009). "Should travelers with hypertension adjust their medications when traveling to high altitude?" High Alt Med Biol 10(1): 11-15.

Luks, A. M. (2016). "Travel to High Altitude Following Solid Organ Transplantation." High Alt Med Biol 17(3): 147-156.

Luks, A. M., R. J. Johnson and E. R. Swenson (2008). "Chronic kidney disease at high altitude." J Am Soc Nephrol 19(12): 2262-2271.

Muxfeldt, E. S., C. R. Cardoso and G. F. Salles (2009). "Prognostic value of nocturnal blood pressure reduction in resistant hypertension." Arch Intern Med 169(9): 874880.

Parati, G., G. Bilo, A. Faini, B. Bilo, M. Revera, A. Giuliano, C. Lombardi, G. Caldara, F. Gregorini, K. Styczkiewicz, A. Zambon, A. Piperno, P. A. Modesti, P. Agostoni and G. Mancia (2014). "Changes in $24 \mathrm{~h}$ ambulatory blood pressure and effects of angiotensin II receptor blockade during acute and prolonged high-altitude exposure: a randomized clinical trial." Eur Heart J 35(44): 3113-3122.

Redon, J., V. Oliver, M. D. Zaragoza and M. J. Galindo (1999). "Ambulatory blood pressure during diseases of the kidney." Blood Press Monit 4(5): 267-274. Suh, K.-S., T. Kim, N.-J. Yi and G. Hong (2015). "Preparation for high altitude expedition and changes in cardiopulmonary and biochemical laboratory parameters with ascent to high altitude in transplant patients and live donors." Clinical Transplantation 29(11): 1013-1020.

van Adrichem, E. J., M. J. Siebelink, B. L. Rottier, J. M. Dilling, G. Kuiken, C. P. van der Schans and E. A. Verschuuren (2015). "Tolerance of Organ Transplant Recipients to Physical Activity during a High-Altitude Expedition: Climbing Mount Kilimanjaro." PLoS One 10(11): e0142641.

Velasquez, M. T., S. Beddhu, E. Nobakht, M. Rahman and D. S. Raj (2016).

"Ambulatory Blood Pressure in Chronic Kidney Disease: Ready for Prime Time?" Kidney international reports 1(2): 94-104. 
Wang, C., Z. Ye, Y. Li, J. Zhang, Q. Zhang, X. Ma, H. Peng and T. Lou (2016). "Prognostic Value of Reverse Dipper Blood Pressure Pattern in Chronic Kidney Disease Patients not Undergoing Dialysis: Prospective Cohort Study." Sci Rep 6: 34932.

White, M., R. Touyz, Y. Tessier, V. Van Le, H. Ross and M. G. Sirois (2009). "A cardiac and a kidney transplant patient above 6000 meters in Bolivia." Wilderness Environ Med 20(1): 99-100. 